\title{
Single-dose pharmacokinetic study of green tea catechins: impact of catechol-O-methyltransferase genotype
}

\author{
R. J. Moore ${ }^{1}$, K. G. Jackson ${ }^{1}$, A. L. Brown' ${ }^{2}$, A. E. Mayes ${ }^{2}$, B. Nicol' ${ }^{2}$, T. Dadd $^{2}$ and A. M. Minihane \\ ${ }^{1}$ Department of Food and Nutritional Sciences, University of Reading, RG6 6AP, UK and ${ }^{2}$ Unilever Discover, \\ Colworth Science Park, MK44 ILQ, UK
}

The biopotency of green tea has been attributed to its polyphenol content, with (-)-epigallocatechin-3-gallate (EGCG) being the most abundant green-tea polyphenol followed by (-)-epigallocatechin (EGC), (-)-epicatechin (EC) and epicatechin-3-gallate (ECG). However, the extent of green-tea polyphenol biotransformation may affect its proposed therapeutic effects. The enzyme responsible for polyphenolic methylation, catechol-O-methyl transferase (COMT), has a common polymorphism which results in an amino acid change at position 158 in the protein proposed to result in a $40 \%$ reduction in enzyme activity ${ }^{(1,2)}$. A pilot study was designed to investigate the impact of the COMT Val158Met genotype on green-tea catechin metabolism.

Twenty healthy participants (10 of each homozygous COMT genotype) were recruited and blood samples were taken at baseline and $30,60,90,120,150,180,240,360$ and $480 \mathrm{~min}$ after the consumption of the capsules containing $1.1 \mathrm{~g}$ Sunphenon decaffeinated green-tea extract $(880 \mathrm{mg}$ green-tea catechins). A low-flavanoid breakfast [2372.328 kJ (567 kcal), $107 \mathrm{~g}$ carbohydrate, $18.1 \mathrm{~g}$ protein and $7.4 \mathrm{~g}$ fat] and a sandwich lunch $[2765.624 \mathrm{~kJ}(661 \mathrm{kcal}), 74.4 \mathrm{~g}$ carbohydrate, $15.8 \mathrm{~g}$ protein and $33.3 \mathrm{~g}$ fat] were given 60 and $240 \mathrm{~min}$ after the capsules. Green-tea catechins were measured by HPLC with coulometric array electrochemical detection and UPLC-Q-TOF Mass Spectrometry.

BMI, weight, total cholesterol, TAG and age were not different between groups. Plasma pharmacokinetic profiles were produced for EGCG, EGC, EC and 4'OMethylEGCG. For the group as a whole, EGCG, EGC, EC and 4'OMethylEGCG reached maximum concentrations of 499.8, 124.2, 96.1 and $36.4 \mathrm{ng} / \mathrm{ml}$ at $81.5,98.5,99.0$ and $96.5 \mathrm{~min}$, respectively. Bactrian curves were observed for the non-gallated plasma green-tea catechins EC and EGC as opposed to single-peaked curves for the gallated plasma green-tea catechins EGCG and ECG (Fig. 1). No significant parametric differences were found between genotype groups in relation to EGCG methylation or effect on EGCG metabolism.
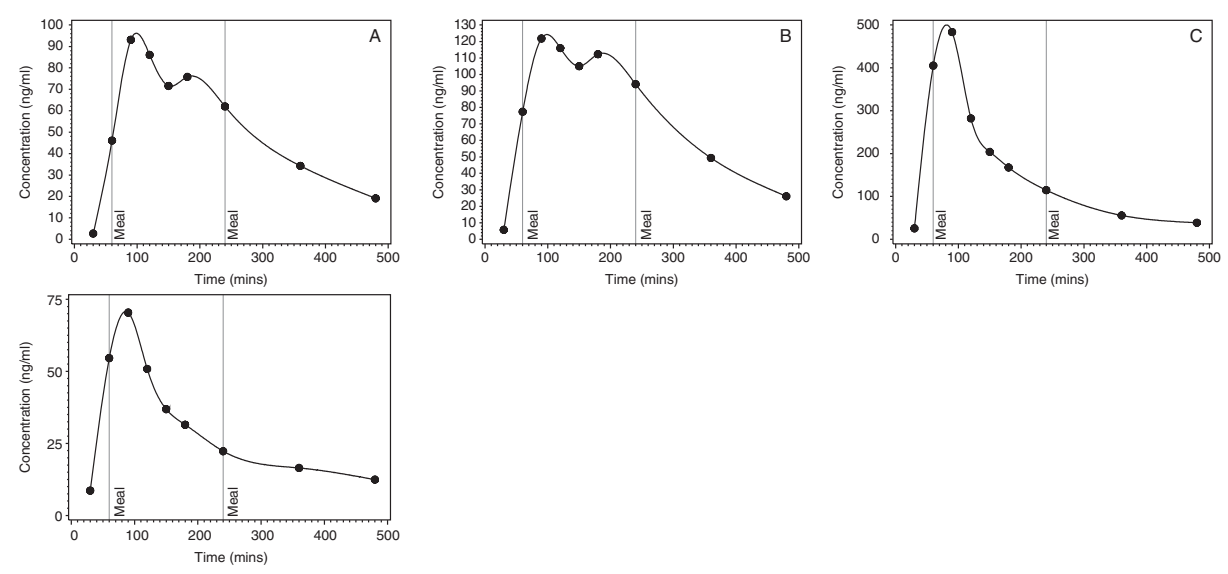

Fig. 1. Pharmacokinetic profiles (A) EC, (B) EGC, (C) EGCG, (D) ECG in the group as a whole after a single dose of $1.1 \mathrm{~g}$ green-tea extract.

In conclusion, this study demonstrated a possible difference in non-gallated and gallated catechin absorption when a meal is consumed after capsule administration. Differences in green-tea catechin metabolism between COMT genotypes were not found, suggesting that genotypic variation has little impact on green-tea catechin metabolism.

1. Chen J et al. (2004) Am J Hum Genet 75, 807-821.

2. Shield AJ, Thomae BA, Eckloff BW et al. (2004) Mol Psychiatry 9, 151-160. 